La Revue

des Droits

de l'Homme

\section{La Revue des droits de l'homme}

Revue du Centre de recherches et d'études sur les droits fondamentaux

Actualités Droits-Libertés | 2008

\title{
Protection des données personnelles(Biométrie)
}

Droit à la vie privée (Art. 8 CEDH)

\section{Sylvia Preuss-Laussinotte}

\section{OpenEdition}

\section{Journals}

Édition électronique

URL : http://journals.openedition.org/revdh/3546

DOI : $10.4000 /$ revdh.3546

ISSN : 2264-119X

\section{Éditeur}

Centre de recherches et d'études sur les droits fondamentaux

\section{Référence électronique}

Sylvia Preuss-Laussinotte, "Protection des données personnelles(Biométrie) », La Revue des droits de I'homme [En ligne], Actualités Droits-Libertés, mis en ligne le 05 décembre 2008, consulté le 01 mai 2019. URL : http://journals.openedition.org/revdh/3546; DOI : 10.4000/revdh.3546

Ce document a été généré automatiquement le 1 mai 2019.

Tous droits réservés 


\title{
Protection des données personnelles(Biométrie)
}

Droit à la vie privée (Art. 8 CEDH)

\author{
Sylvia Preuss-Laussinotte
}

1 La Cour européenne des droits de l'homme a rendu en Grande Chambre un arrêt très attendu, puisqu'elle statuait sur la question de l'enregistrement de données biométriques relatives à des condamnations dans des bases de données au Royaume-Uni : empreintes digitales et données génétiques. Le Royaume-Uni se désigne lui-même comme « précurseur » en matière de données biométriques, puisqu'il a notamment la plus grande banque de données génétiques européennes; mais il «est aussi le seul Etat membre à autoriser expressément la conservation systématique et illimitée à la fois des profils et des échantillons relatifs aux personnes condamnées.»(\$48) Les requérants se plaignaient, sous l'angle des articles 8 et 14 de la Convention, de ce que les autorités avaient conservé leurs empreintes digitales, échantillons cellulaires et profils génétiques après la conclusion, par un acquittement et par une décision de classement sans suite, des poursuites pénales menées contre eux. Toutes leurs démarches visant à l'effacement de ces données avaient été rejetées. Tout en reconnaissant une "légère atteinte à la vie privée ", la Chambre des Lords avait estimé que "l'intérêt de conserver les empreintes digitales et les échantillons recueillis sur les suspects était considérable ».Si les requérants soutenaient que la conservation de leurs échantillons faisaient peser sur eux des soupçons alors qu'ils avaient été acquittés, le ministre de l'intérieur soutenait que « pareille conservation n'avait rien à voir avec le passé, c'est-à-dire avec l'infraction dont la personne avait été acquittée, mais visait à faciliter les enquêtes sur des infractions futures ».

2 La Cour rappelle que la notion de « vie privée » est « une notion large, non susceptible d'une définition exhaustive, qui recouvre l'intégrité physique et morale de la personne (...) Elle peut donc englober de multiples aspects de l'identité physique et sociale d'un individu.(...)Le simple fait de mémoriser des données relatives à la vie privée d'un individu constitue une ingérence au sens de l'article $8(\$ 67)$, position qu'elle a déjà largement développé en autonomisant la notion de données personnelles dans le cadre de l'article $8 \mathrm{CEDH}$. Elle fait par ailleurs référence aux 
textes spécifiques de protection des données personnelles, dont la Convention sur la protection des données du Conseil de l'Europe STE 108 de 1981.

3 Affirmant que les trois catégories d'informations personnelles conservées par les autorités au sujet des deux requérants, à savoir des empreintes digitales, des profils ADN et des échantillons cellulaires, « constituent toutes des données à caractère personnel au sens de la Convention sur la protection des données car elles se rapportent à des individus identifiés ou identifiables ", la Cour analyse ensuite les différentes données biométriques, notamment les profils ADN qui «fournissent un moyen de découvrir les relations génétiques (et ethniques) pouvant exister entre des individus (ce qui) suffit en soi pour conclure que leur conservation constitue une atteinte au droit à la vie privée de ces individus». Elle applique le même raisonnement aux empreintes digitales numérisées, notant qu'elles «constituent des données à caractère personnel (...) et qu'elles contiennent certains traits externes d'identification, tout comme, par exemple, des photographies ou des échantillons de voix. » Leur enregistrement constitue là aussi une atteinte au droit à la vie privée.

C'est sur le plan plus précis de la protection de ces données à caractère personnel que la Cour va constater la violation de l'article 8 , dans la mesure où cette protection «joue un rôle fondamental pour l'exercice du droit au respect de la vie privée et familiale consacré par l'article 8 de la Convention. La législation interne doit donc ménager des garanties appropriées pour empêcher toute utilisation de données à caractère personnel qui ne serait pas conforme aux garanties prévues dans cet article ». Elle observe que « la protection offerte par l'article 8 de la Convention serait affaiblie de manière inacceptable si l'usage des techniques scientifiques modernes dans le système de la justice pénale était autorisé à n'importe quel prix et sans une mise en balance attentive des avantages pouvant résulter d'un large recours à ces techniques, d'une part, et des intérêts essentiels s'attachant à la protection de la vie privée, d'autre part (...) Cour considère que tout Etat qui revendique un rôle de pionnier dans l'évolution de nouvelles technologies porte la responsabilité particulière de trouver le juste équilibre en la matière(\$ 112)

5 En conclusion, la Cour estime que «le caractère général et indifférencié du pouvoir de conservation des empreintes digitales, échantillons biologiques et profils ADN des personnes soupçonnées d'avoir commis des infractions mais non condamnées (...) ne traduit pas un juste équilibre entre les intérêts publics et privés concurrents en jeu, et que l'Etat défendeur a outrepassé toute marge d'appréciation acceptable en la matière. Dès lors, la conservation litigieuse s'analyse en une atteinte disproportionnée au droit des requérants au respect de leur vie privée et ne peut passer pour nécessaire dans une société démocratique ( $\$ 125$ ). Cette décision est particulièrement intéressante au moment où les Etats surenchérissent en créant des banques de données biométriques de plus en plus importantes, et en mettant en place dans le cadre du traité de Prüm un échange de ces données désormais facilité. Rappelons qu'en France, les profils ADN peuvent être conservés pendant vingt-cinq ans après un acquittement ou l'abandon des poursuites. Si le procureur de la République peut ordonner leur suppression avant l'expiration de ce délai, «soit d'office soit sur demande si la conservation n'est plus nécessaire à des fins d'identification dans le cadre de poursuites pénales ", force est de constater que cette suppression est extrêmement rare: outre le fait que la procédure ouverte aux personnes est peu connue, la notion de nécessité de conservation à des fins d'identification est conçue de manière très extensive. 
S. et Marper c. Royaume-Uni (requêtes $n^{\text {os }}$ 30562/04 et 30566/04), 4 décembre 2008

\section{AUTEUR}

\section{SYLVIA PREUSS-LAUSSINOTTE}

Professeure de droit public à l'Université de Paris Nanterre 